\title{
Immunological aspects of antitumor photodynamic therapy outcome
}

\author{
MAEGORZATA WACHOWSKA ${ }^{l}$, ANGELIKA MUCHOWICZ ${ }^{2}$ URSZULA DEMKOW
}

${ }^{1}$ Department of Laboratory Diagnostics and Clinical Immunology of Developmental Age, Medical University of Warsaw, Warsaw, Poland ${ }^{2}$ Department of Immunology, Center of Biostructure Research, Medical University of Warsaw, Warsaw, Poland

\begin{abstract}
Photodynamic therapy (PDT) of cancer is an efficient and promising therapeutic modality approved for the treatment of several types of tumors and non-malignant diseases. It involves administration of a non-toxic photosensitizer followed by illumination of the tumor site with a harmless visible light. A light activated photosensitizer can transfer its energy directly to molecular oxygen, leading to production of highly toxic reactive oxygen species (ROS). Antitumor effects of PDT result from the combination of three independent mechanisms involving direct cytotoxicity to tumor cells, destruction of tumor vasculature and induction of the acute local inflammatory response. PDT-mediated inflammatory reaction is accompanied by tumor infiltration of the leukocytes, enhanced production of pro-inflammatory factors and cytokines. Photodynamic therapy is able to effectively stimulate both the innate and the adaptive arm of the immune system. In consequence, this regimen can lead to development of systemic and specific antitumor immune response. However, there are limited studies suggesting that under some specific circumstances, PDT on its own may exert some immunosuppressive effects leading to activation of immunosuppressive cells or cytokines production. In this report we briefly review all immunological aspects of PDT treatment.
\end{abstract}

Key words: cancer, antitumor immunity, photodynamic therapy.

(Cent Eur J Immunol 2015; 40 (4): 481-485)

\section{Introduction}

Photodynamic therapy (PDT) is a successful and minimally invasive therapeutic approach used in the treatment of various solid tumors as well as non-malignant diseases. Photodynamic therapy has been clinically approved for the treatment of lung, esophageal, bladder, skin and head and neck cancers. Photodynamic therapy action is dependent on three essential components: photosensitizer that is applied topically or administered systemically; visible light, usually generated by laser sources; and molecular oxygen. None of these elements is toxic, only when combined together they exert a cytotoxic effect to tumor cells. In a clinical setting PDT is a two-step procedure involving administration of a photosensitizing agent that preferentially accumulates in the tumor tissue, followed by local irradiation of the lesion with the light of appropriate wavelength able to activate the sensitizer [1]. The light activated photosensitizer can either transfer its energy directly to molecular oxygen, leading to production of highly reactive singlet oxygen, or can react with macromolecules and generate radicals. Further reaction with molecular oxygen results in formation of superoxide ion, hydroxyl radical or hydrogen peroxide. All these reactive oxygen species
(ROS) are responsible for oxidative damage of proteins, lipids and other intracellular molecules, causing PDT-mediated direct tumor cell death through apoptosis, necrosis or autophagy [2]. Antitumor effects of PDT result from its unique mechanism involving not only direct cytotoxicity to tumor cells, but also indirect actions such as disruption of tumor vasculature and induction of acute local inflammatory response (Fig. 1). The latter may facilitate the development of antitumor and antigen-specific immune response [3].

\section{Induction of inflammatory response}

It was widely reported that PDT treatment leads to oxidative stress in tumor tissue associated with massive photooxidative damage in cancer cells, tumor vasculature and stroma. This PDT-mediated injury is a local trauma and threat for tissue integrity and homeostasis. Therefore, host inflammatory response is elicited in order to remove dead and damaged cells, heal injured tissue and restore its function as well as the disrupted homeostasis [4]. Oxidative stress caused by PDT may induce various signals transduction in the cell which are responsible for production of stress-induced proteins, activation of genes regulating apop-

Correspondence: Małgorzata Wachowska, PhD, Department of Laboratory Diagnostics and Clinical Immunology of Developmental Age, Medical University of Warsaw, Marszalkowska 24, 00-576 Warsaw, Poland, e-mail: malgorzata.wachowska@wum.edu.pl 


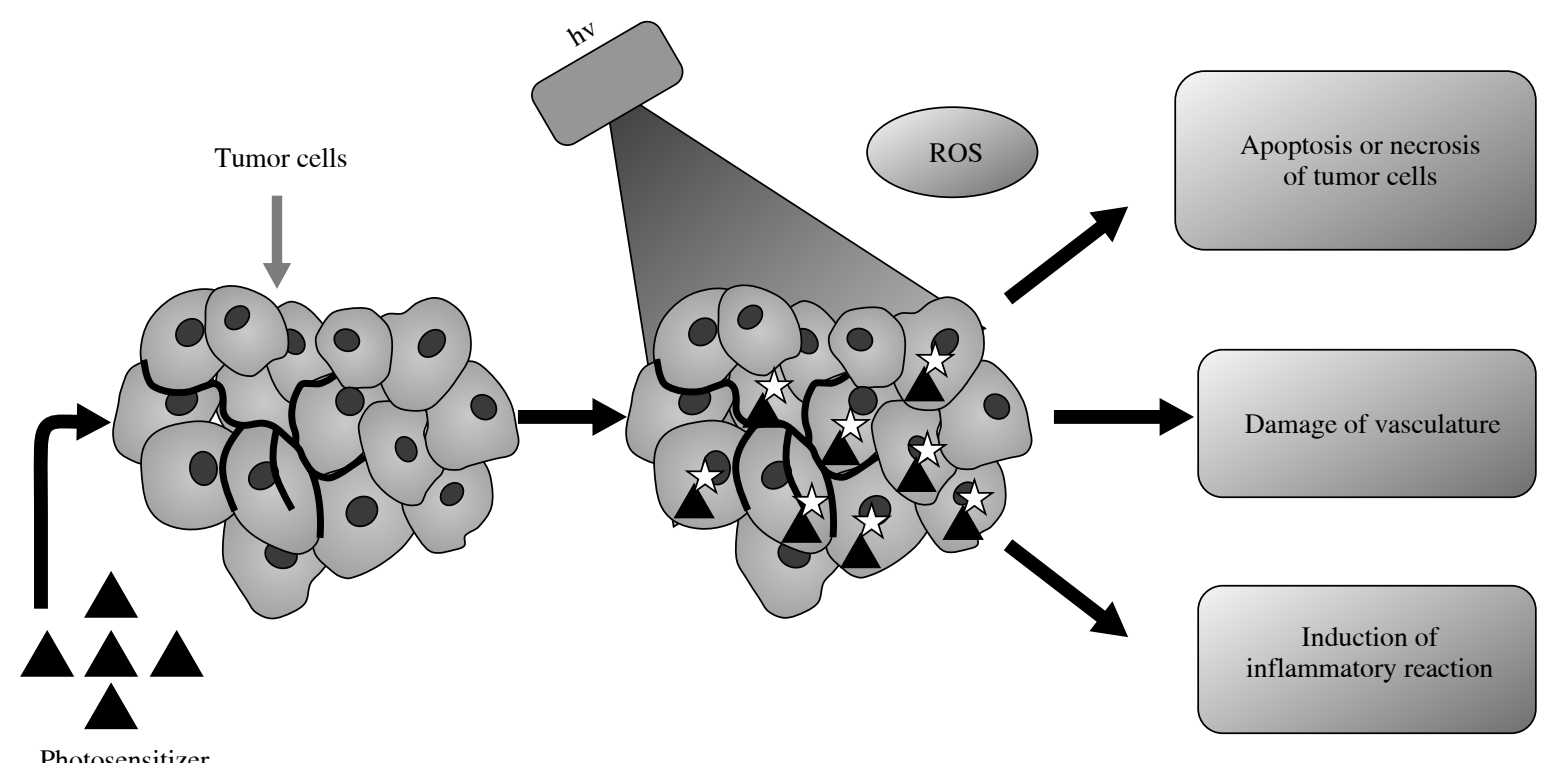

Photosensitizer

Fig. 1. Unique mechanism of PDT action

tosis and cytokine gene expression. Photooxidative damage of cell membranes results from peroxidation of lipids that leads to extensive release of arachidonic acid metabolites which are potent inflammatory mediators [5]. Importantly, similar effects are encountered during infection or other tissue injury. Additionally, another source of inflammatory signals are vascular events induced by PDT. PDT-mediated damage of endothelial cells causes disruption of their barrier function, loss of junctions, and exposure of the vascular basement membrane. Exposure of sites enabling clot formation initiates clotting cascade, activation of platelets and the release of proaggregatory and vasoactive agents. This is followed by vessel contraction which facilitates leucocytes and thrombocytes adhesion, whereas released mediators increase vessel permeability. These events result in venules occlusion and blood flow stasis.

The process of inflammation is one of the most essential mechanisms in antitumor action of PDT. This inflammatory reaction is mediated by various factors such as vasoactive substances, components of the complement and clotting cascades, acute phase proteins, proteinases, peroxidases, ROS, leukocyte chemoattractants, cytokines, growth factors and other immunoregulators [6].

\section{Innate arm of immune response}

PDT-induced damage of tumor and stroma cells leads directly to an increased level of cytokines and other mediators of inflammation. Further release of cytokines is a consequence of activation of various signaling pathways and transcription factors such as activator protein 1 (AP-1) or nuclear factor $\kappa \mathrm{B}(\mathrm{NF}-\kappa \mathrm{B})$ [7]. After PDT treatment in animal and human studies, elevated levels of numerous cytokines such as interleukin (IL)-1 $\beta$, IL-2, IL-6, IL-10, tumor necrosis factor $\alpha$ (TNF- $\alpha$ ) were confirmed. However, it seems that the most important cytokine in PDT outcome is IL-1 $\beta$. There are studies showing that IL-1 $\beta$ activity is critical for the therapeutic efficacy, since its neutralization reduces the cure rates of PDT-treated tumors in a mouse model. Interestingly, blocking of anti-inflammatory cytokines such as IL-10 and TGF- $\beta$ improved the cure rates of PDT-treated tumors [4].

Release of immunomodulating factors together with acute phase response from PDT-injured tissue is followed by rapid and massive accumulation of various immune cells into the tumor site. The infiltrating cells mainly comprise neutrophils, but also mast cells and monocytes/ macrophages [8]. Shortly after PDT, neutrophils massively migrate to the tumor site and orchestrate other immune cells and further development of immune response. There is increasing evidence that neutrophils are indispensable for PDT efficacy. Depletion of these cells in tumor bearing mice and rats led to attenuation of PDT-mediated destruction of tumor cells. Furthermore, macrophages/monocytes are also considered as effector cells in the elimination of PDT-treated tumor tissue. A number of studies indicate that low dose PDT can stimulate macrophages, leading to enhanced phagocytic activity and release of TNF- $\alpha$ and nitric oxide (NO). Moreover, tumoricidal activity of these cells was confirmed by in vivo studies showing that stimulation of macrophages potentiates antitumor effects of PDT, whereas inactivation of macrophages causes decreases in cure rates in mice [9]. Another class of non-specific immune cells contributing to the antitumor effect of 
PDT is NK cells. It was reported that depletion of NK cells in a mouse model leads to abrogated antitumor response towards distant lesions, indicating that NK cells play a pivotal role in PDT outcome [10].

One of the earliest events in PDT-treated cells is massive release of various natural and oxidatively modified tumour antigens together with cell stress factors known as damage-associated molecular patterns (DAMPs) [11]. DAMPs are intracellular molecules, which act as immunostimulators when exposed on the surface or released by damaged and/ or dying cells [4]. DAMPs are molecules recognized by the innate immune system through pattern recognition receptors (PRR) [12]. It has been shown that PDT enhances the immunogenicity of dead tumor cells by inducing release of DAMPs and tumor antigens. Altogether, tumor antigens and DAMPs with other pro-inflammatory signals can activate both innate and adaptive immune response [13].

\section{Adaptive arm of immune response}

Dendritic cells (DCs) are the most potent antigen presenting cells (APCs) that link innate and adaptive immune response. Dendritic cells capture and recognize antigens, become activated and home to local lymph nodes, where they mature and present antigen-derived peptides in association with major histocompatibility complex (MHC) molecules to $\mathrm{T}$ lymphocytes. This results in activation of CD4+ T helper cells and CD8+ cytotoxic T cells, $B$ cells and initiation of the adaptive immune response [14]. The pro-inflammatory PDT effect, together with released tumor antigens and danger signals, enhances maturation and activation of DCs and further stimulation of $\mathrm{T}$ cell effector functions [15]. It was reported that immature DCs injected into PDT-treated tumors can capture tumor antigens, become mature and migrate into draining lymph nodes, where they stimulate specific T cells [16]. During an effective immune response, activated T lymphocytes become effector T cells and migrate to the lesion and kill the tumor cells.

The role of the immune system in PDT outcome has been broadly investigated for the last decades indicating that antitumor effects of PDT depend on the presence and activity of adaptive immunity [17]. Korbelik et al. reported long-term cures in BALB/c mice inoculated with EMT6 mammary carcinoma cells, whereas the same PDT regimen was not able to cure SCID mice. Notably, adoptive transfer of splenocytes obtained from previously cured mice fully restored the curative effect of PDT [18]. Interestingly, there are also human studies reporting that PDT is effective in achieving local and distant tumor control. Thong et al. describes a case report of a patient with recurrent angiosarcoma. The main cluster of tumors on the right upper limb was treated with Fotolon-PDT. Unexpectedly, 2 months after therapy, the untreated tumors on the same limb underwent spontaneous remission. Similarly, 4 months after treatment spontaneous remission of the untreated tumors on the other limb was observed. Sustained remission of these tumors was achieved for 15 months after PDT. Moreover, biopsy of the irradiated site revealed strong infiltration of CD4+T lymphocytes and subsequent massive accumulation of CD8+ T lymphocytes [19].

\section{Immunosuppressive effect of photodynamic therapy}

Activation of antitumor immune response by PDT is of great interest, whereas little attention is paid to the immunosuppressive side of PDT. PDT-mediated tissue damage causes release of various agents leading to activation of immune response. Every strong immune activation induces counteraction such as immunosuppressive mechanisms in order to restore homeostasis and prevent from dangerous over-active immune responses. Therefore, it is not surprising that there are studies reporting induction of immunosuppression by PDT [20]. Elmets and Bowen were the first to report immunosuppressive effects of PDT. Treatment of mice with hematoporphyrin derivative (HpD) PDT resulted in 50\% suppression of contact hypersensitivity (CHS) to 2,4-di-nitrofluorobenzene (DNFB) [21]. Subsequently, it was shown that PDT can lead to systemic immunosuppression that can be adoptively transferred by macrophages [22].

PDT-activated DCs transport tumor antigens to the LNs not only to stimulate but also to inhibit immune response. The function of activated T cells might be prevented by regulatory T cells (Tregs) or immunosuppressive cytokines such as IL-10 or transforming growth factor $\beta$ (TGF- $\beta$ ) that are elevated in response to PDT treatment. Those cytokines stimulate CD4+ T cells to differentiate into Tregs, leading to generation of anergic or tolerogenic CD8+ T cells [23]. Recently, it has been reported that the level of Tregs is significantly elevated in spleens and lymph nodes (LNs) in a tumor bearing mouse after PDT treatment [24].

Additionally, PDT-induced ROS production possibly may cause inactivation of immunogenic molecules, such as DAMPs, released after PDT [25]. Moreover, enhanced production of vascular endothelial factor c, VEGFc, after PDT treatment might affect the maturation process of DCs that results in development of tolerogenic and immunosuppressive environment [26].

Mechanisms of PDT-induced immunosuppression is still poorly understood and mostly tested in the CHS model. Therefore, PDT-mediated immunosuppression in the tumor setting requires further investigations.

\section{Summary}

Ideal anticancer therapy would lead to eradication of not only the primary tumor, but also induce specific antitumor immune response allowing for the control of distant 
metastases and protection from tumor relapse. It seems that PDT may meet these expectations as it induces acute inflammation, attracts immune cells to distant tumors and is able to develop systemic antitumor immune response. Under certain experimental settings, PDT itself can lead to complete regression of tumor and to development of long-lasting tumor-specific immunity. However, such situations are unusual; in most cases PDT alone is insufficient in inducing immune response that would lead to complete tumor rejection. Nevertheless, antitumor PDT creates a unique microenvironment for further development of effective antitumor immune response. Therefore, PDT with its unique features gives new possibilities for combination treatments, especially with drugs stimulating immune response. There are several studies reporting enhanced anti-tumor immune response when PDT is combined with immune-stimulating agents such as recombinant cytokines [granulocyte-colony stimulating factor (G-CSF), granulocyte-macrophage colony-stimulating factor (GM-CSF) and TNF]; epigenetic drug, 5-aza-2'-deoxycytidine or intratumoral administration of DCs [8, 16, 27-29].

On the other hand, PDT can induce immunosuppression leading to activation of anti-inflammatory cytokines and suppressing immune cells. Consequently, combination therapies inhibiting suppressive environment should also be taken under consideration. It was widely reported that neutralization of anti-inflammatory cytokines such as IL-10 or TGF- $\beta$ improved PDT outcome. Moreover, application of PDT combined with cyclophosphamide led to depletion of Tregs and enhanced PDT-mediated immunity as well as to long-term survival and development of memory immunity in mice [24].

To summarize, all those events involved in immune response against tumors induced by PDT should be further studied. Understanding of these mechanisms will allow for rational design of combination therapies that could be applied in clinical settings.

\section{References}

1. Agostinis P, Berg K, Cengel KA, et al. (2011): Photodynamic therapy of cancer: an update. CA Cancer J Clin 4: 250-281.

2. van Duijnhoven FH, Aalbers RI, Rovers JP, et al. (2003): The immunological consequences of photodynamic treatment of cancer, a literature review. Immunobiology 2: 105-113.

3. Nowis D, Makowski M, Stokłosa T, et al. (2005): Direct tumor damage mechanisms of photodynamic therapy. Acta Biochim Pol 2: 339-352.

4. Korbelik M (2006): PDT-associated host response and its role in the therapy outcome. Lasers Surg Med 5: 500-508.

5. Korbelik M, Dougherty GJ (1999): Photodynamic therapy-mediated immune response against subcutaneous mouse tumors. Cancer Res 8: 1941-1946.

6. Dolmans DE, Fukumura D, Jain RK (2003): Photodynamic therapy for cancer. Nat Rev Cancer 5: 380-387.

7. Kick G, Messer G, Goetz A, et al. (1995): Photodynamic therapy induces expression of interleukin 6 by activation of AP-1 but not NF-kappa B DNA binding. Cancer Res 11: 2373-2379.

8. Krosl G, Korbelik M, Krosl J, Dougherty GJ (1996): Potentiation of photodynamic therapy-elicited antitumor response by localized treatment with granulocyte-macrophage colony-stimulating factor. Cancer Res 14: 3281-3286.

9. Canti G, De Simone A, Korbelik M (2002): Photodynamic therapy and the immune system in experimental oncology. Photochem Photobiol Sci 1: 79-80.

10. Belicha-Villanueva A, Riddell J, Bangia N, Gollnick SO (2012): The effect of photodynamic therapy on tumor cell expression of major histocompatibility complex (MHC) class I and MHC class I-related molecules. Lasers Surg Med 1: 60-68.

11. Firczuk M, Nowis D, Golab J (2011): PDT-induced inflammatory and host responses. Photochem Photobiol Sci 5: 653663.

12. Krysko DV, Agostinis P, Krysko O, et al. (2011): Emerging role of damage-associated molecular patterns derived from mitochondria in inflammation. Trends Immunol 4: 157-164.

13. Garg AD, Nowis D, Golab J, et al. (2010): Immunogenic cell death, DAMPs and anticancer therapeutics: an emerging amalgamation. Biochim Biophys Acta 1: 53-71.

14. Wachowska M, Gabrysiak M, Muchowicz A, et al. (2014): 5-Aza-2'-deoxycytidine potentiates antitumour immune response induced by photodynamic therapy. Eur J Cancer 7: 1370-1381.

15. Gollnick SO, Brackett CM (2010): Enhancement of anti-tumor immunity by photodynamic therapy. Immunol Res 1-3: 216-226.

16. Jalili A, Makowski M, Switaj T, et al. (2004): Effective photoimmunotherapy of murine colon carcinoma induced by the combination of photodynamic therapy and dendritic cells. Clin Cancer Res 13: 4498-4508.

17. Korbelik M, Krosl G, Krosl J, et al. (1996): The role of host lymphoid populations in the response of mouse EMT6 tumor to photodynamic therapy. Cancer Res 24: 5647-5652.

18. Castano AP, Mroz P, Hamblin MR (2006): Photodynamic therapy and anti-tumour immunity. Nat Rev Cancer 7: 535-545.

19. Thong PS, Ong KW, Goh NS, et al. (2007): Photodynamic-therapy-activated immune response against distant untreated tumours in recurrent angiosarcoma. Lancet Oncol 10: 950-952.

20. Mroz P, Hamblin MR (2011): The immunosuppressive side of PDT. Photochem Photobiol Sci 5: 751-758.

21. Elmets CA, Bowen KD (1986): Immunological suppression in mice treated with hematoporphyrin derivative photoradiation. Cancer Res 4 Pt 1: 1608-1611.

22. Lynch DH, Haddad S, King VJ, et al. (1989): Systemic immunosuppression induced by photodynamic therapy (PDT) is adoptively transferred by macrophages. Photochem Photobiol 4: 453-458.

23. Griffith TS, Kazama H, VanOosten RL, et al. (2007): Apoptotic cells induce tolerance by generating helpless CD8+ T cells that produce TRAIL. J Immunol 5: 2679-2687.

24. Reginato E, Mroz P, Chung H, et al. (2013): Photodynamic therapy plus regulatory $\mathrm{T}$-cell depletion produces immunity against a mouse tumour that expresses a self-antigen. $\mathrm{Br}$ J Cancer 8: 2167-2174.

25. Kazama H, Ricci JE, Herndon JM, et al. (2008): Induction of immunological tolerance by apoptotic cells requires caspase-dependent oxidation of high-mobility group box-1 protein. Immunity 1: 21-32. 
26. Laxmanan S, Robertson SW, Wang E, et al. (2005): Vascular endothelial growth factor impairs the functional ability of dendritic cells through Id pathways. Biochem Biophys Res Commun 1: 193-198.

27. Golab J, Wilczynski G, Zagozdzon R, et al. (2000): Potentiation of the anti-tumour effects of Photofrin-based photodynamic therapy by localized treatment with G-CSF. $\mathrm{Br}$ J Cancer 8: 1485-1491.

28. Bellnier DA (1991): Potentiation of photodynamic therapy in mice with recombinant human tumor necrosis factor-alpha. J Photochem Photobiol B 2: 203-210.

29. Wachowska M, Gabrysiak M, Muchowicz A, et al. (2014): 5-Aza-2'-deoxycytidine potentiates antitumour immune response induced by photodynamic therapy. Eur J Cancer 2014; 50: 1370-1381. 